treatment. Forming a sexual identity is a key developmental task during this phase, thus understanding the factors that enable the information needs of AYA to be appropriately identified and met is critical. This study expands on previous findings that AYA exhibited significant unmet needs around information provision on sex, body image, and relationships and aims to explore information and support preferences regarding where, how, and from whom AYA want to receive this information.

Methods Four young people aged 24-26 years, with a previous cancer diagnosis at age 14-23 years attended an indepth four-hour workshop. Participatory activities were employed to identify the questions young people had; their support/information preferences; and, their definition of intimacy. The framework approach was used to analyse workshop transcripts.

Results Young people's preferences of information/support provision varied. They wanted trusted sources of information and while some preferred to have face-to-face discussions with healthcare professionals, others felt embarrassed and would prefer an online platform. All acknowledged that listening to peers with cancer talk about sexuality and relationships would 'normalise' their questions. Feeling embarrassed (professionals and young people) was a barrier to an open discussion about their needs. Triggers for information and support included: partners (pressures/expectations); lack of sexual drive (managing side effects); body image concerns (weight gain/loss); physical constraints; and fertility concerns. Young people wanted access to information tailored to their needs.

Conclusion Findings from this study show cancer-specific sex, relationship and body image issues faced by AYA and provide important direction to the development of interventions - a balance between professional and peer support is needed. Sexual health is a key element of wellbeing and a failure to address it may place AYA at risk for long-term consequences related to sexual, identity and relationships development. This study highlights the importance of conversations relating to sexual consequences of illness and age-appropriate assessment and interventions in other contexts where disease may disrupt young people's development.

\section{P54 RISK BEHAVIOR AND PSYCHOLOGICAL WELL-BEING OF LEFT-BEHIND ADOLESCENTS IN TWO PROVINCES OF CHINA}

${ }^{1}$ WM Yuan, ${ }^{1,2}$ T Hesketh*. 'Global Health Centre, Zhejiang University, Hangzhou, China; ${ }^{2}$ Institute for Global Health, University College London, London, UK

10.1136/bmjpo-2019-RCPCH-SAHM.58

Aims In China around 40 million children and adolescents are left behind in rural areas by parents who migrate to cities to work. This study aimed to investigate the effects of prolonged separation from parents, and different guardianship models, on engagement in risk behaviors and psychological well-being.

Methods A cross-sectional study was conducted in migrantsending areas of three counties in Zhejiang province and two in Guizhou in 2015. A self-completion questionnaire was administered among adolescents age 6 to 16 from 56 primary and middle schools, selected through random stratified sampling. Children's demographic characteristics, family and social support, risk behaviors, and psychological wellbeing (using the Strengths and Difficulty Scale) were measured.Logistic regression was used to measure the effect of guardianship types on behavioral and psychological problems.

Results There were 1447 respondents in Zhejiang, and 1773 in Guizhou. The mean age was 12 (SD 2.2). 1238 children were living with both parents, and 1977 were left behind children (LBC). Of these 1025 lived with grandparents, 838 with one-parent and 114 with 'others' (relatives, siblings, alone). After controlling for economic status, age and gender, LBC living with others reported higher prevalence of smoking $(\mathrm{aOR}=2.01$, 95\%CI:1.15-3.51), skipping class $(2.28,1,28$ $4.06)$ and cheating $(2.23,1.55-3.50)$ than non-LBC, but there were no significant differences in drinking, going to internet cafés, stealing, bullying and fighting. Among three types of LBC guardianship, adolescents living with one parent reported lowest level of risk behaviors, and those living with 'others' highest. In addition, LBC living with grandparents reported more emotional problems $(1.25,1.02-1.53)$, LBC living with others more hyperactivity $(2.15,1.41-3.26)$ and fewer peer problems $(0.65,0.43-0.98)$, but there were no significant differences in conduct problems. Risk factors for LBC's behavioral and psychological problems were: living in Guizhou, poor study performance, having friends involved in risky behaviors and low social support.

Conclusion Living with parents or one parent led to more favorable behavioral and psychological outcomes. Migrant parents should try to ensure that one of them stays at home to give care and guidance on a daily basis.

\section{P55 MOVING ON: FROM POND TO SEA - PROVISION OF TRANSITION SERVICES FOR YOUNG PEOPLE WITH NEURO-DEVELOPMENTAL CONDITIONS}

SS Patwardhan*, R Singh. Department of Paediatrics, Glangwili General Hospital, Hywel Dda University Health Board, Carmarthen, UK

\subsection{6/bmjpo-2019-RCPCH-SAHM.59}

Introduction 'Transition' is the purposeful, planned process that addresses medical, psychosocial and educational needs of Young people with long-term conditions as they move from child-centred to adult-oriented healthcare systems. The importance and challenges of healthcare transition are recognised in UK National Institute for Health and Clinical Excellence (NICE) Guideline and Quality Standard (2016). A new dedicated transition clinic for Young people with neuro-developmental problems was initiated in Carmarthenshire in September 2016

Aims To demonstrate the provision of transition services for children with neuro-developmental conditions; benchmark services against NICE standards and illustrate areas for improvement.

Methods Young people aged 16-19 years who were seen in a transition clinic with diagnosis of neuro-developmental problems in Carmarthenshire. 50 Young people from September 2016 to July 2018 were reviewed.

Results 38 were male and 12 were female. Diagnosis were ADHD 40\%, Learning difficulties 34\%, ASD 24\%, Developmental coordination disorder 16\%, Physical disability 14\%. 